\title{
ENERGY EXTRACTION FOR THE LHC SUPERCONDUCTING CIRCUITS
}

\author{
K. Dahlerup-Petersen, F. Rodriguez-Mateos, R. Schmidt, F. Sonnemann \\ CERN, CH-1211 Geneva 23, Switzerland
}

\begin{abstract}
The superconducting magnets of the LHC will be powered in about 1700 electrical circuits. The energy stored in circuits, up to $1.3 \mathrm{GJ}$, can potentially cause severe damage of magnets, bus bars and current leads. In order to protect the superconducting elements after a resistive transition, the energy is dissipated into a dump resistor installed in series with the magnet chain that is switched into the circuit by opening current breakers.

Experiments and simulation studies have been performed to identify the LHC circuits that need energy extraction. The required values of the extraction resistors have been computed. The outcome of the experimental results and the simulation studies are presented and the design of the different energy extraction systems that operate at $600 \mathrm{~A}$ and at $13 \mathrm{kA}$ is described.
\end{abstract}

\section{INTRODUCTION}

The magnet system for the LHC includes about 9000 magnets that are powered in more than 1700 electrical circuits.

In each sector of the LHC that corresponds to $1 / 8$ of the machine, three circuits operating at a current of up to $13 \mathrm{kA}$ power the main bending magnets (MB), the main focusing and the main defocusing quadrupole magnets (MQ). A single magnet is de-coupled from the other magnets in the circuit by diodes installed parallel to each magnet inside the helium enclosure. Quench heaters are mounted on the magnet coils that are fired after detection of a resistive transition, in order to provoke a quench of a large fraction of the coil. The stored energy is then better distributed and excessive temperatures and voltages are avoided. In order to extract the energy of the entire circuit, dump resistors are switched in series with the magnets. The energy extraction system is also protecting the superconducting bus bars interconnecting the magnets in case of a bus bar quench.

Depending on the parameters of a circuit such as stored energy, operating current, magnet and bus bar parameters, different types of energy extraction systems have been developed [1].

\section{ENERGY EXTRACTION SYSTEMS}

\subsection{Energy Extraction for the main circuits}

The LHC machine will require thirty-two energy extraction systems for its twenty-four $13 \mathrm{kA}$ main power circuits. The energy of a circuit with the main bending magnets will have two identical energy extraction systems, one in the mid-point of the electrical circuit and one close to the power converter. Each of the eight QF and eight QD main quadrupole circuits will have a single extraction system. The 32 installations consist of more than $300 \mathrm{t}$ of equipment, including heavy extraction resistors, high-current DC breakers and drivers together with the control electronics. The principal components are being supplied through collaboration agreements with Institutes in CERN non-member States [2].

The main parameters for one MB circuit are:

- Number of dipoles: 154

- Inductance: $16.0 \mathrm{H}$

- Stored magnetic energy at a current of $13 \mathrm{kA}$ : $1353 \mathrm{MJ}$

- Extraction resistance: 2 x $75 \mathrm{~m} \Omega$

- Initial time constant: $107 \mathrm{~s}$

- Maximum current decay rate during extraction: $-122 \mathrm{~A} / \mathrm{s}$

- Maximum voltage to ground during extraction: $488 \mathrm{~V}$.

The main parameters for one MQ circuit are (either MQF or MQD):

- Number of quadrupoles: 47 or 51

- Inductance: $263 \mathrm{mH} / 285 \mathrm{mH}$

- Stored magnetic energy at a current of $13 \mathrm{kA}: 23 \mathrm{MJ} /$ $27 \mathrm{MJ}$

- Extraction resistance: $6.6 \mathrm{~m} \Omega / 7.7 \mathrm{~m} \Omega$

- Initial time constant: $40 \mathrm{~s} / 38 \mathrm{~s}$

- Maximum current decay rate during extraction: $-340 \mathrm{~A} / \mathrm{s}$

- Maximum voltage to ground during extraction: $100 \mathrm{~V}$.

The fundamental choices of circuit topology, system configuration as well as rating and selection of components are based on experience from a number of pilot systems, built for LHC magnet test benches at CERN over a period of almost ten years.

For the dipole circuits, a series-connected topology was chosen. The extraction switches with their parallel dump resistors are inserted in series with the magnets. This allows insertion of more, symmetrically placed systems. The maximum voltage to ground is reduced by a factor of four with respect to the total system voltage $(1950 \mathrm{~V})$ as the earth connection is placed in the electrical centre of the dump resistor that is close to the power converter. This configuration limits the size of the large dump 
resistors since they need to absorb only half of the stored energy. The chosen dump resistance is a compromise between the energy deposition in the cold diode, which will conduct the current in the event of a quench during the discharge, and the maximum admissible current decay rate to avoid magnetic quench-back.

Each component is designed for high reliability and long life expectancy. Semiconductor switches were discarded because of their limited radiation resistance, their high on-state losses and because they rely on a single, active opening trigger. Electro-mechanical breakers can be designed with two independent release mechanisms (pulsed trigger and under-voltage release), the losses are typically 10 times lower than an equivalent thyristor switch and they can be installed in the LHC tunnel radiation field. A total number of 280 fast DC breaker units, with a current rating of $4 \mathrm{kA}$, are required for the main circuits in the LHC machine. They were developed in collaboration with Russian Industry, with the driver electronics designed by CERN. They have no mechanical "catch-and-latch" and are based on a unique three-limb magnetic circuit, combining closing / holding and the fast and slow opening features. The breaker array consists of four parallel branches with each having two series-connected switches for redundancy. A power busway of quasi-coaxial structure assures a correct current distribution between the branches.

The extraction resistors have a forced-air cooled, selfinductance compensated stainless steel body and a built-in air-to-water heat exchanger. Each $75 \mathrm{~m} \Omega$ unit for the dipole circuit consists of three individual, parallelconnected modules of $4.5 \mathrm{t}$. They are designed for installation in the tunnel below the beam pipes. During the energy deposition, the resistor body temperature rises up to $350{ }^{\circ} \mathrm{C}$. The cooling period represents typically 90 minutes. For the quadrupole chains the dump resistor is a single unit housed in a cubicle. Presently, pre-series components are undergoing severe testing and the system is being subjected to a global risk analysis and endurance testing [2].

\subsection{Energy Extraction for the 600 A circuits}

The main parameters are:

- Total number of circuits with energy extraction: 176

- Circuit families: MCS, MCD, MO, MQT-MQS, MQT, MQTL, MS

- Total circuit inductance: $12-720 \mathrm{mH}$

- Stored energy: $2-73 \mathrm{~kJ}$

- Discharge time constants: $20-1030 \mathrm{~ms}$

- Extraction resistance: $0.2 \Omega$ and $0.7 \Omega$.

The concept is based on two, series-connected, fast, mechanical AC breakers, specially equipped with DC arc shutes. The three, simultaneously operated poles are parallel-connected to carry the 600 ADC. Snubber capacitors are used for arc limitation, reduction of contact erosion and shortening of the total opening time $(17 \mathrm{~ms})$.
Air convection cooled, non-inductive dump resistors of alloy $\mathrm{FeCrAl}$ or stainless steel are switched into the circuit for the extraction. Typical cooling time is 30 minutes [3].

\subsection{Energy Extraction by the Power Converter}

For circuits of very low voltage and small stored energy, the energy extraction system will be a part of the power converter. Such systems consist of a combination of a semi-conductor crowbar and a low-voltage switch. The additional switch is required since an over-voltage during a quench could create a short-circuit in the output stage of the converter, which would render the crowbar inoperative. In total 160 circuits will be equipped with this type of extraction [4].

\section{SIMULATION STUDIES}

Simulations were carried out to study whether energy extraction facilities are required to protect either magnets and/or bus bars. Two simulation programs have been developed.

SPQR (Simulation Program for Quench Research), based on the finite difference method, approximates numerically the heat balance equation including the cooling conditions and gives the quench propagation as well as the hot spot temperatures [5].

In order to simulate the voltage and temperature development of a series of magnets the simulation package QUABER has been developed at CERN [6], based on the commercial tool SABER ${ }^{\mathrm{TM}^{*}}$. The QUABER libraries require quench parameters as input data that can be provided by experimental results or by SPQR simulation results.

The outcome of the simulation studies has been compared with experimental results from tests on individually powered magnets. Since the experimental verification for an entire magnet circuit is not possible before LHC commissioning, the simulation model was extended to predict the necessity to install energy extraction facilities for the various LHC superconducting circuits. The objective of the studies was to determine the circuits that require energy extraction facilities to limit the number of extraction facilities to a safe minimum. The results are summarised in Table 1 [7]. The voltage applied when opening the breaker system is shown in column 5. In column 6 the value of the resistor of the extraction system is shown, required to keep temperatures within safe limits. A single quenching magnet is "de-coupled" from other magnets in the circuit by using a resistor that is mounted parallel to the quenching magnet (similar to the diode across the main magnets). This avoids the dissipation of the full energy stored in the circuit into the quenching magnet. In some cases such resistor will be installed since energy extraction alone is not sufficient to avoid overheating of the coils. This is shown in column 7 of the

\footnotetext{
* SABER is a Trademark of Analogy INC.
} 


\begin{tabular}{|c|c|c|c|c|c|c|c|}
\hline $\begin{array}{c}(1) \\
\text { Magnet } \\
\text { family }\end{array}$ & $\begin{array}{c}(2) \\
\text { Current } \\
{[\mathrm{A}]}\end{array}$ & $\begin{array}{c}(3) \\
\text { Magnets } \\
\text { per circuit }\end{array}$ & $\begin{array}{c}(4) \\
\text { Energy } \\
\text { extraction }\end{array}$ & $\begin{array}{c}(5) \\
\mathrm{U}_{\mathrm{ex}} \\
{[\mathrm{V}]}\end{array}$ & $\begin{array}{c}(6) \\
\mathrm{R}_{\mathrm{ex}}[\mathrm{m} \Omega]\end{array}$ & $\begin{array}{c}(7) \\
\mathrm{R}_{\mathrm{par}}[\mathrm{m} \Omega]\end{array}$ & Reason for energy extraction \\
\hline MB & 11810 & 154 & yes & 1950 & 150 & diode & busbar \\
\hline MQ & 11810 & 47 or 51 & Yes & 100 & $6.6 / 7.7$ & diode & busbar \\
\hline MCS & 550 & 154 & yes & 420 & 700 & 80 & magnet and busbars \\
\hline MCD & 550 & 77 & yes & 420 & 700 & - & magnet and busbar \\
\hline MS & 550 & 12 & yes & 420 & 700 & 150 & magnet and busbar \\
\hline MS & 550 & 10 & yes & 420 & 700 & 150 & magnet and busbar \\
\hline MSS & 550 & 4 & yes & 30 & 50 & 150 & busbar \\
\hline MQT & 550 & 8 & yes & 420 & 700 & 250 & magnet and busbar \\
\hline MQT & 550 & 1 & yes & 30 & 50 & - & busbar \\
\hline MQS & 550 & 4 & yes & 30 & 50 & 250 & busbar \\
\hline MQS & 550 & 2 & yes & 30 & 50 & - & magnet and busbar \\
\hline MQTL & 400 & 6 & yes & 420 & 700 & 200 & magnet and busbar \\
\hline MQTL & 550 & 2 & yes & 420 & 700 & 200 & magnet and busbar \\
\hline MQTL & 550 & 1 & yes & 30 & 50 & - & magnet and busbar \\
\hline MO & 550 & 13 & yes & 120 & 200 & - & magnet \\
\hline MO & 550 & 8 & yes & 120 & 200 & - & magnet \\
\hline
\end{tabular}

Table 1: The various circuits requiring energy extraction. $R_{\mathrm{ex}}$ is the required value for the resistance in the extraction facility, $R_{\mathrm{par}}$ is the required value for the parallel resistor across the magnet.

table $\left(R_{\mathrm{par}}\right.$ is the value of the parallel resistor required). For some of the circuits the energy needs to be extracted if a bus bar would quench (see column 8) even if no protection would be required in case of a magnet quench.

Where possible, low-voltage extraction facilities are installed in the power converters, allowing for reduction of costs and space in the busy zones where this equipment will be located in the LHC machine.

\section{DATABASE}

The requirements for energy extraction have been included in the LHC Powering Database for reference during the installation period.

\section{CONCLUSIONS}

The energy extraction facilities required for the safe protection of the superconducting elements of the LHC electrical circuits of 0.6 and $13 \mathrm{kA}$ rating have been determined in the light of simulations and experimental data. A major step towards the final qualification of this equipment will be achieved after tests and operation at the new LHC full-cell prototype (String-2) installed in CERN, where first units of the two types of facilities have been installed and commissioned.

\section{ACKNOWLEDGEMENTS}

The authors are thankful to F. Bordry from the Power Converter Group at CERN for his commitment to the success of this work. Without the distinct and precise advice from K. H. Mess (DESY) this task would not have been completed. Thanks also to P. Proudlock and N. Siegel for their encouragement.

\section{REFERENCES}

[1] K. Dahlerup-Petersen et al., "The Protection System for the Superconducting Elements of the Large Hadron Collider at CERN", PAC'1999, New York, USA.

[2] K. Dahlerup-Petersen et al., "Energy Extraction Resistors for the Main Dipole and Quadrupole Circuits of the LHC', EPAC'2000, Vienna, Austria.

[3] K. Dahlerup-Petersen, private communication.

[4] EEWG, LHC Powering Workshop, CERN, 2000. http://hcp.web.cern.ch/lhcp/TCC/PLANNING/TCC/ Power2000/welcome.htm

[5] F. Sonnemann and R. Schmidt, "Quench simulations of superconducting elements in the LHC accelerator", Cryogenics 40 (2000) p519-529.

[6] D. Hagedorn and F. Rodriguez-Mateos, "Modelling of the quenching process ...", IEEE Trans. Magn. Vol 28, January 1992.

[7] F. Sonnemann, "Resistive Transition and Protection of LHC Superconducting Cables and Magnets", PhD Thesis, University of Aachen-CERN, February 12, 2001. 